Poznańskie Studia Teologiczne 29(2015), s. 91-104.

doi: $10.14746 /$ pst.2015.29.5

Mieczysław Polak ${ }^{1}$

Uniwersytet im. Adama Mickiewicza w Poznaniu

Wydział Teologiczny

\title{
Kairos der Barmherzigkeit. Barmherzigkeit als Prinzip einer missionarischen Seelsorge
}

\section{Einleitung}

Die bisherige Lehre des Papstes Franziskus lässt sich mit einigen Begriffen charakterisieren. $\mathrm{Zu}$ den leitenden Schlüsselworten seines Pontifikats, die er in Bezug auf die Pastoral der Kirche verwendet, gehören zweifellos: missio und misericordia. Das sind zwar keine neuen Begriffe der kirchlichen Lehre, aber sie bekamen durch den Papst Franziskus eine neue Qualität und eine noch größere Bedeutung. Meistens wird der Begriff missio in Zusammenhang mit den Begriffen „Seelsorge“ und „Pastoral“ verwendet. Das „,missionarische Handeln“ ist für Franziskus „das Paradigma für alles Wirken der Kirche“. Der gewünschte Entwicklungsweg der Pastoral sollte wiederum „,von einer rein bewahrenden Pastoral zu einer entschieden missionarischen Pastoral“ führen. Ähnliches gilt es auch für den Begriff misericordia: „Barmherzigkeit ist der letzte und endgültige Akt mit dem Gott uns entgegentritt“ ${ }^{\text {“2 }}$ und „die Kirche muss der Ort der ungeschuldeten Barmherzigkeit sein, wo alle sich aufgenommen und geliebt fühlen können, wo sie Verzeihung erfahren und sich ermutigt fühlen können, gemäß dem guten Leben des Evangeliums zu leben“3. Im vorliegenden Beitrag wird zuerst versucht, das Profil einer missionarischen Seelsorge zu skizieren und die Barmherzigkeit als ein „Zeichen der Zeit“ zu deuten. Diese Überlegungen werden dann dazu dienen, eine neue pastorale Mentalität, die sich auf einer Spiritualität der Barmherzigkeit stützt, zu entwickeln.

\footnotetext{
${ }^{1}$ Prezbiter, dr hab., adiunkt w Zakładzie Teologii Pastoralnej na Wydziale Teologicznym Uniwersytetu im. Adama Mickiewicza w Poznaniu, e-mail: mpolak@amu.edu.pl.

${ }^{2}$ Franziskus, Verkündigungsbulle Misericordiae vultus zum Außerordentlichen Jubiläum der Barmherzigkeit, deutsche Übersetzung, Verlautbarungen des apostolischen Stuhls Bd. 200, hrsg. Sekretariat der Deutschen Bischofskonferenz, Bonn 2015, Nr. 1 (ab hier: MV).

${ }^{3}$ Franziskus, Apostolisches Schreiben Evangelii gaudium über die Verkündigung des Evangeliums in der Welt von heute (24.11.2013), deutsche Übersetzung, Verlautbarungen des Apostolischen Stuhls, hrsg. Sekretariat der deutschen Bischofskonferenz, Bd. 194, Bonn 2014, Nr. 15. 114 (ab hier: EG).
} 


\section{Das Profil einer missionarischen Pastoral}

Die Begriffe wie „Mission“ und „Evangelisierung“ waren in der Vergangenheit häufig negativ besetzt. Man verband mit ihnen Erfahrungen der Intoleranz und des aufdringlichen Bekehrungseifers. Die negativen Assoziationen, die mit diesen Worten identifiziert sind, entstanden vor allem im Hinblick auf den problematischen Zusammenhang von Kolonialisierung und Missionierung ${ }^{4}$. Nicht selten wurde die Evangelisierung bzw. die Neuevangelisierung als „Re-Christianisierung“ oder „Re-Katholisierung“ missverstanden. Man könnte den Eindruck haben, so als ginge es darum, zu einer vergangenen Gestalt des Glaubens zurückzukehren. Darüber hinaus fühlen sich manche auch damit überfordert, den eigenen Glauben anderen gegenüber ins Wort zu bringen, weil sie es sich nicht zutrauen, ,das Zeugnis des Wortes“ angemessen weiterzugeben 5 .

Nicht nur in Westeuropa aber auch in Polen weisen die Zeichen der Zeit immer mehr in die Richtung, dass das konstantinische Bündnis zwischen Kirche und Volk, beziehungsweise Staat immer mehr gelöst wird. $\mathrm{Zu}$ diesem Bündnis gehörte auch die vorgegebene Selbstverständlichkeit des Hineinwachsens der Menschen in die Kirche, was aufgrund ebenso selbstverständlicher Sozialisationspraxis des Glaubens erfolgte. Heutzutage wird sie stets unwirksamer ${ }^{6}$. Nach Knobloch fällt „,die Wieder- bzw. Neuentdeckung der missionarischen Präsenz der Kirche in eine Zeit tiefgreifender aktueller gesellschaftlicher Transformationsprozesse, die sich im Begriff des mehrdimensionalen Globalisierungsphänomens bündeln lassen. Dieses Phänomen kann in den Ortskirchen - darunter verstehen wir Bistümer und Dekanate ebenso wie Pfarrverbände und Gemeinden - zwei Reaktionen hervorrufen: Zum einen die Reaktion des Mitvollzugs der Differenzierungen der Globalisierungsprozesse und der Öffnung auf die mit der Globalisierung gegebenen Herausforderungen, zum anderen die Reaktion der Abschottung, um so die ortskirchliche spirituelle Homogenität und Identität vor der Tsunamiwelle der Globalisierung zu schützen" ${ }^{\text {"7 }}$.

Jedoch die Glaubensüberlieferung bleibt weiterhin eine fortdauernde Aufgabe der Kirche, weil eben der Glaube durch seine Weitergabe stark wird ${ }^{8}$. Die Dynamik dieser Aufgabe gründet auf der „trinitarischen Sendung“ und auf dem missionarischen Auftrag der Kirche. „Der missionarische Impuls - lehrt Johanes

\footnotetext{
${ }^{4}$ Vgl. K. Koch, Mission oder De-Mission der Kirche? Herausforderungen an eine notwendige Neuevangelisierung in: Mission als Herausforderung. Impulse zur Neuevangelisierung, hrsg. G. Augustin, K. Krämer, Freiburg im Breisgau 2011, S. 42.

${ }^{5}$ Vgl. Die Deutsche Bischofskonferenz, „Zeit zur Aussaat“. Missionarisch Kirche sein (26.11.2000), Verlautbarungen der deutschen Bischofskonferenz, hrsg. Sekretariat der deutschen Bischofskonferenz, Bd. 68, Bonn 2000, S. 18.

${ }^{6}$ Vgl. K. Koch, Mission oder De-Mission der Kirche?, op. cit., S. 44.

${ }^{7}$ S. Knobloch, Ungenutztes Potential. Zwischen religiöser Sehnsucht und Kirchenkrise, Ostfildern 2011, S. 85.
} 
Paul II. - ist mithin zutiefst in der Natur des christlichen Lebens verwurzelt und gibt auch der ökumenischen Bewegung ihre Stoßrichtung: »Alle sollen eins sein: Wie du, Vater, in mir bist und ich in dir bin, sollen auch sie in uns sein, damit die Welt glaubt, dass du mich gesandt hast « (Joh 17, 21)“ (RMis 1).

In der Geschichte der Kirche gab es unterschiedliche Formen der Weitergabe des Glaubens. Seit frühmittelalterlichen Zeiten herrschte eine „soziale“ Form vor, die sich seit dem Beginn der Reformationszeit in eine ,pädagogische“ Form der Glaubensvermittlung weiterentwickelte. Jetzt aber treten wir ,in eine Zeit ein, in der christlicher Glaube missionarisch-evangelisierend in der Generationenabfolge weitergegeben werden muss"'.

Seit dem Zweiten Vatikanischen Konzil fanden die lehramtlichen Stellungsnahmen zur Missionsaufgabe der Kirche in vielen offiziellen Verlautbarungen ihren Ausdruck. Zuerst aber lässt sich die Allgegenwart des Missionsthemas in den Dokumenten des Zweiten Vatikanischen Konzils leicht beobachten. Das Konzil selbst äußerte sich in beinahe allen Konstitutionen, Dekreten und Erklärungen zur missionarischen Aufgabe der Kirche. Diese Stellungnahme hat ihren Grund darin, dass das Konzil den Missionsauftrag der Kirche in den gesamten Heilsplan Gottes mit der Menschheit eingeordnet hat und dass man schon vor dem Konzil von einigen Hauptstädten oder christlichen Ländern sagte, sie seien „Missionsländer“ geworden ${ }^{10}$.

Die nachkonziliaren Päpste sind nicht müde geworden, den Missionsauftrag der Kirche in die Mitte der kirchlichen Identität zu stellen. In den Dokumenten wie: Apostolisches Schreiben Evangelii nuntiandii vom Paul VI., Enzyklika Redemptoris missio und Apostolisches Schreiben Novo millennio ineunte vom Johannes Paul II. und Enzyklika Deus caritas est vom Bendedikt XVI. befinden sich die grundsätzlichen Impulse für die missionarische Erneuerung des kirchlichen Lebens und Wirkens. Paul VI. betrachtet die Evangelisierung als „die Gnade und eigentliche Berufung der Kirche, ihre tiefste Identität"11. Im Mittelpunkt der missionarischen Erneuerung der Kirche steht in der Sicht Papst Paul VI. die Kategorie des Zeugnisses, ,und zwar in der sensiblen Wahrnehmung, dass der heutige Mensch keine Lehrer, sondern Zeugen braucht, und Lehrer insofern, als sie auch als Zeugen wahrgenommen werden können“ (vgl. EN 41) ${ }^{12}$.

\footnotetext{
${ }^{8}$ Vgl. Johannes Paul II., Enzyklika Redemptoris missio über die fortdauernde Gültigkeit des missionarischen Auftrages (7.12.1990), deutsche Übersetzung, Verlautbarungen des Apostolischen Stuhls, hrsg. Sekretariat der deutschen Bischofskonferenz, Bd. 100, Bonn 1991, Nr. 2 (ab hier: RMis).

${ }^{9}$ Die Deutsche Bischofskonferenz, „Zeit zur Aussaat“, op. cit., S. 33-34.

${ }^{10}$ K. Koch, Mission oder De-Mission der Kirche?, op. cit., S. 52.

${ }^{11}$ Vgl. Paul VI., Apostolisches Schreiben Evangelii nuntiandi über die Evangelisierung in der Welt von heute (8.12.1975), deutsche Übersetzung, Verlautbarungen des Apostolischen Stuhls, hrsg. Sekretariat der deutschen Bischofskonferenz, Bd. 2, Bonn 1975, Nr. 14 (ab hier: EN).

${ }^{12}$ K. Koch, Mission oder De-Mission der Kirche?, op. cit., S. 52.
} 
Obwohl die Päpste im Bezug auf die traditionell christlichen Länder eher den Begriff „Neuevagelisierung“ verwenden, stellt Johannes Paul II. fest, dass es auch in traditionell christlichen Ländern, Gegenden, und Menschengruppen nicht evangelisierte Bereiche gibt, die der speziellen Leitung der Mission ad gentes anvertraut sind. „Es ist also auch in diesen Ländern nicht nur eine Neu-Evangelisierung, sondern in einigen Fällen eine erstmalige Evangelisierung geboten“" RMis 37).

Die vom Papste Benedikt XVI. einberufene Bischofsynode zum Thema: Die neue Evangelisierung für die Weitergabe des christlichen Glaubens sollte dazu beitragen, dass die Kirche mit erneuertem Eifer ihre eigene Sendung zur Evangelisierung übernimmt. In der Lineamenta der XIII. Ordentlichen Vollversammlung der Bischofssynode ${ }^{13}$ befanden sich Fragen, die auf Antworten von unterschiedlichen kirchlichen Gremien warten. Eine von ihnen lautet: „Ist die Dringlichkeit einer neuen, missionarischen Verkündigung ein gewohnter Bestandteil der pastoralen Tätigkeit der Gemeinschaften geworden?"14. „Missionarische Verkündigung“, „missionarische Seelsorge“, „missionarischer Sinn der kirchlichen Tätigkeiten“ das sind Termine, die nach Lineamenta den Charakter der gegenwärtigen Pastoral bestimmen sollen. Sie beinhalten einen Aufruf zur ,pastoralen Umkehr“ christlicher Gemeinschaften ${ }^{15}$.

Als das nachsynodale Dokument erschien das Apostolische Schreiben vom Papst Franziskus, das Evangelii gaudium betitelt wurde. Sehr deutlich und emotional drückt Franziskus in diesem Dokument die Notwendigkeit des missionarischen Profils der Pastoral der Kirche aus. „Ich träume - schreibt er - von einer missionarischen Entscheidung, die fähig ist, alles zu verwandeln, damit die Gewohnheiten, die Stile, die Zeitpläne, der Sprachgebrauch und jede kirchliche Struktur ein Kanal werden, der mehr der Evangelisierung der heutigen Welt als der Selbstbewahrung dient. Die Reform der Strukturen, die für die pastorale Neuausrichtung erforderlich ist, kann nur in diesem Sinn verstanden werden: dafür zu sorgen, dass sie alle missionarischer werden, dass die gewöhnliche Seelsorge in all ihren Bereichen expansiver und offener ist, dass sie die in der Seelsorge Tätigen in eine ständige Haltung des »Aufbruchs « versetzt und so die positive Antwort all derer begünstigt, denen Jesus seine Freundschaft anbietet" (EG 27).

Eine missionarische Umgestaltung bildet für Franziskus den zentralen Punkt der pastoralen Umkehr der Kirche und ihr erstes Anliegen, weil die Missionstätigkeit auch heute noch die größte Herausforderung für die Kirche darstellt. Der Erneuerungsweg der Kirche soll ,,von einer rein bewahrenden Pastoral zu einer entschieden missionarischen Pastoral übergehen“ und das missionarische Han-

${ }^{13}$ XIII. Ordentliche Vollversammlung der Bischofssynode, Neuevangelisierung für die Weitergabe des Glaubens, Lineamenta. Text: http://www.vatican.va/roman_curia/synod/documents/rc_syn od_doc_20110202_lineamenta-xiii-assembly_ge.html [zugriff am 15.06.2015]

${ }^{14}$ Ebd. Nr. 17.

${ }^{15}$ Vgl. Ebd. Nr. 10. 
deln soll als „das Paradigma für alles Wirken der Kirche“ erkannt werden (vgl. EG 15). Franziskus führt den Missionsauftrag der Kirche auf seinen Ursprung zurïck, nämlich auf den Auftrag, der von Jesus den Zwölf gegeben wurde „Geht hin in alle Welt und verkündet das Evangelium“ (Mk 16,15). In ihm ist eine „Dynamik des Aufbruchs“ tief verankert. „Heute sind in diesem Geht Jesu die immer neuen Situationen und Herausforderungen des Evangelisierungsauftrags der Kirche gegenwärtig, und wir alle sind zu diesem neuen missionarischen „Aufbruch“ berufen. Jeder Christ und jede Gemeinschaft soll unterscheiden, welches der Weg ist, den der Herr verlangt, doch alle sind wir aufgefordert, diesen Ruf anzunehmen: hinauszugehen aus der eigenen Bequemlichkeit und den Mut zu haben, alle Randgebiete zu erreichen, die das Licht des Evangeliums brauchen“ (EG 20).

\section{Barmherzigkeit als ,Zeichen der Zeit"}

In einem Interview für die Jesuitenzeitschrift „La Civiltà Cattolica” sagte Papst Franziskus: „Mit großer Klarheit sehe ich, dass die Kirche heute am meisten die Fähigkeit benötigt, die Wunden zu heilen, die Herzen der Gläubigen zu wärmen, Nähe zu erweisen. Ich vergleiche die Kirche mit einem Feldspital nach einer Schlacht. Es ist nicht zielführend, einen Schwerverletzten nach seinem Cholesterinspiegel oder Blutzucker zu fragen! Man muss seine Wunden heilen"16. Die biblische Tradition verbindet die Heilung der Wunden mit der Barmherzigkeit (vgl. Lk 10,30-37). Eine Pastoral der Barmherzigkeit ist also ein Weg der Kirche, auf dem ein Prozess der Heilung der Wunden in Gang gesetzt werden soll. Der päpstliche Ruf nach Barmherzigkeit findet große Resonanz in der Seelsorge. Dennoch stellt W. Kasper fest, dass ,dieser Begriff oft verkommen und herabgekommen ist zu einer «soften» Pastoral und Spiritualität und zu einer blutund kraftlosen Weichheit, der jede Entschiedenheit und jedes klare Profil abgeht, die es nur jedem irgendwie recht machen will“"17.

Vier Päpste der zweiten Hälfte des 20. Jahrhunderts und vom Beginn des 21. Jahrhunderts - Johannes XIII., Johannes Paul II., Benedikt XVI. und Franziskus - stellen Barmherzigkeit in die Mitte der kirchlichen Verkündigung. Insbesondere jedoch ist die Barmherzigkeit ein zentrales Thema der Lehre von Johannes XXIII., Johannes Paul II. und Franziskus ${ }^{18}$. Für Johannes XXIII. stellt die Barmherzigkeit „den schönsten Namen und die schönste Anrede Gottes“ dar. In seiner

\footnotetext{
${ }_{16}$ Text: http://pl.radiovaticana.va/news/2013/09/19/papie\%C5\%BC_udzieli\%C5\%82 jezuitom_ obszernego_wywiadu_o_sobie,_ko\%C5\%9Bciele_i/pol-730040 [zugriff am: 03.11.2014].

${ }^{17}$ W. Kasper, Barmherzigkeit. Grundbegriff $\bar{d}$ es Evangeliums - Schlüssel christlichen Lebens, Freiburg im Breisgau 2012, S. 20.

${ }^{18}$ Vgl. ebd., S. 15-19.
} 
Rede zur Eröffnung des Zweiten Vatikanischen Konzils stellte er fest, dass sich die Kirche in ihrer Verkündigung lange Zeit auf die Verurteilung der Irrtümer konzentrierte. Heute aber „möchte die Braut Jesu Christi lieber das Heilmittel der Barmherzigkeit anwenden als die Waffe der Strenge erheben"19. Die konziliare Reflexion über die Verkündigung und das Leben der Kirche, die Johannes XXIII. entscheidend geprägt hat, regte einen neuen pastoralen Still an. „Der neue pastorale Stil, den Johannes XXIII. meinte, hat viel mit dem zu tun, was er in seiner Eröffnungsrede mit dem Wort vom Heilmittel der Barmherzigkeit angesprochen hat. Seither ist das Thema Barmherzigkeit grundlegend geworden, nicht nur für das Konzil, sondern für die ganze pastorale Praxis der nachkonziliaren Kirche“"20.

Die Botschaft von der Barmherzigkeit Gottes war auch für Johannes Paul II. das Leitthema seines Pontifikats ${ }^{21}$. Die folgenden wichtigsten Ereignissen sind hier zu erwähnen: Enzyklika Dives in missericordia über das göttliche Erbarmen $^{22}$, die dem Thema Barmherzigkeit gewidmet war ${ }^{23}$; die erste Heiligsprechung im dritten Jahrtausend, durch die der Papst die mystische Erfahrung von der Barmherzigkeit Gottes der bis dahin nur wenig bekannten polnischen Ordensschwester Faustyna Kowalska der ganzen Kirche als Geschenk Gottes an unsere Zeit vorstellte; die Erklärung den zweiten Sonntag in der Osterzeit zum „Sonntag der Barmherzigkeit" zu ernennen, was auf Anregung von Schwester Faustyna erfolgte ${ }^{24}$. Aber nicht so viel durch seine Lehre und symbolische Taten sondern vielmehr durch das Zeugnis seines Leidens verkündete Johannes Paul II. die Botschaft von der Barmherzigkeit Gottes. Diese Botschaft hat er der Kirche des 21. Jahrhunderts ,ins Stammbuch geschrieben“25.

\footnotetext{
${ }^{19}$ Zitiert nach: W. Kasper, Barmherzigkeit, op. cit., S. 16.

${ }^{20}$ W. Kasper, Barmherzigkeit, op. cit., S. 16.

${ }^{21}$ Vgl. Johannes Paul II, Erinnerung und Identität, deutsche Übersetzung, Augsburg 2005, S. 75.

22 Johannes Paul II, Enzyklika Dives in missericordia über das göttliche Erbarmen (30.11.1980), deutsche Übersetzung, Verlautbarungen des Apostolischen Stuhls, hrsg. Sekretariat der deutschen Bischofskonferenz, Bd. 26, Bonn 1980 (ab hier: DiM).

${ }^{23} \mathrm{Zu}$ deisem Thema: S. Grzybek, M. Jaworski, Encyklika Ojca Świętego Jana Pawła II o Bożym miłosierdziu „Dives in missericordia”. Tekst i komentarz, Kraków 1981; Jan Pawet II „Dives in missericordia”. Tekst $i$ komentarze, hrsg. S. Nagy, Lublin 1983; J. Bajda, Encyklika o Bożym mitosierdziu, „Collectanea Theologica” 4(1981), S. 85-88; Wobec tajemnicy Bożego Miłosierdzia, hrsg. L. Balter, Reihe: Powołanie człowieka, Poznań 1991, S. 27-37; A. Gajda, Miłosierdzie większe niż sprawiedliwość. Piękno myśli i słowa w encyklice „,Dives in missericordia”, „Zeszyty Naukowe KUL" 3(2004), S. 85-101; Johannes Paul II, Barmherzigkeit Gottes - Quelle der Hoffnung, ausgewählt und eingeleitet von E. Olk, Einsideln 2011; P.M. Zulehner, Gott ist größer als unser Herz. Eine Pastoral des Erbarmens, Ostfildern 2006, S. 25-28.

${ }^{24}$ Vgl. Johannes Paul II, Predigt bei der Heiligsprechung von Maria Faustyna Kowalska, Łagiewniki 30.04.2000, Text: http://w2.vatican.va/content/john-paul-ii/de/homilies/2000/docu ments/hf_jp-ii_hom_20000430_faustina. html [zugriff am 03.07.2015]. Die Lehre von Johannes Paul II. über die Barmherzigkeit Gottes bezieht sich primär nicht auf die Privatoffenbarung von der hl. Sr. Faustyna, wie das P.M. Zulehner behauptet. Vgl. P.M. Zulehner, Kirchenvisionen. Orientierung in Zeiten des Kirchenumbaus, Ostfildern 2012, S. 67.
}

${ }^{25}$ W. Kasper, Barmherzigkeit, op. cit. S. 17. 
Den Aufschrei der Kirche um Gottes Erbarmen bezieht Johannes Paul II. auf die Lage der Welt von heute, in der unterschiedliche Umwandlungen stattfinden, nicht nur solche, ,die zur Hoffnung auf eine bessere Zukunft des Menschen auf dieser Erde berechtigen, sondern auch vielfache Bedrohungen, welche über die bisher gekannten weit hinausgehen" (vgl. DiM 2). Die Veränderungen verursachen im modernen Menschen eine innere Unruhe und eine existentielle Angst. Johannes Paul II. diagnostizierte diese Situation auf folgende Weise: „Der zeitgenössische Mensch fühlt diese Drohungen. (...) Der Mensch von heute stellt sich oft die angsterfüllte Frage nach der Lösung der entsetzlichen Spannungen, die sich über der Welt zusammengeballt haben und das Leben der Menschen durchziehen. Und wenn er manchmal nicht den Mut hat, das Wort »Erbarmen" auszusprechen, oder in einem areligiösen Bewusstsein auch kein entsprechendes findet, muss es die Kirche um so nachdrücklicher aussprechen, nicht nur in ihrem eigenen Namen, sondern auch im Namen aller Menschen von heute" (DiM 15) ${ }^{26}$.

Papst Benedikt XVI. setzte den neuen pastoralen Stil in der Reflexion und in der Praxis fort. In seinen Äußerungen nach dem Tod von Johannes Paul II. zeigte er deutlich darauf hin, dass das „Evangelium der Barmherzigkeit“ das größte pastorale Anliegen seines Vorgängers war. In der Predigt während der Messe für die Wahl des neuen Papstes hat er sich auf die Lehre seines unlängst verstorbenen Vorgängers sehr deutlich bezogen: ,Jesus Christus ist die göttliche Barmherzigkeit in Person: Christus begegnen heißt, der Barmherzigkeit Gottes begegnen. (...) Die Barmherzigkeit Christi ist keine billig zu habende Gnade, sie darf nicht als Banalisierung des Bösen missverstanden werden. Christus trägt in seinem Leib und in seiner Seele die ganze Last des Bösen, dessen ganze zerstörerische Kraft. Er verbrennt und verwandelt das Böse im Leiden, im Feuer seiner leidenden Liebe“ ${ }^{\text {227. }}$.

Eine theologisch vertiefte und den Herausforderungen der gegenwärtigen ,Zeichen der Zeit" entsprechende Konzeption der Barmherzigkeit Gottes stellte Papst Benedikt XVI. in seinen Enzykliken Deus caritas est $t^{28}$ und Caritas in veritate ${ }^{29}$ dar. In diesen Dokumenten stellt er die Liebe in den Mittelpunkt, und sie ist für ihn das

\footnotetext{
${ }^{26}$ Vgl. M. Polak, Duszpasterstwo miłosierdzia. Miłosierdzie jako paradygmat pastoralnej misji Kościoła, in: „Teologia Praktyczna” 15(2014), S. 8-9.

${ }^{27}$ J. Ratzinger, Predigt während der Messe pro eligendo romano Pontifice. Patriarchalbasilika St. Peter 18.04.2005. Text: http://www.vatican.va/gpII/documents/homily-pro-eligendo-pontifice_ 20050418_ge.html [zugriff am 03.07.2015].

${ }^{28}$ Benedikt XVI, Enzyklika Deus caritas est über die christliche Liebe (25.12.2005), deutsche Übersetzung, Verlautbarungen des apostolischen Stuhls Bd. 171, hrsg. Sekretariat der Deutschen Bischofskonferenz, Bonn 2006 (ab hier: DCE).

${ }^{29}$ Benedikt XVI, Enzyklika Caritas in veritate über die ganzheitliche Entwicklung des Menschen in der Liebe und in der Wahrheit (29.06.2009), deutsche Übersetzung, Verlautbarungen des apostolischen Stuhls Bd. 186, hrsg. Sekretariat der Deutschen Bischofskonferenz, Bonn 2009 (ab hier: $\mathrm{CiV})$.
} 
Grundparadigma der kirchlichen Soziallehre: „Die Liebe geht über die Gerechtigkeit hinaus, denn lieben ist schenken, dem anderen von dem geben, was «mein» ist; aber sie ist nie ohne die Gerechtigkeit, die mich dazu bewegt, dem anderen das zu geben, was «sein» ist, das, was ihm aufgrund seines Seins und seines Wirkens zukommt. Ich kann dem anderen nicht von dem, was mein ist, «schenken», ohne ihm an erster Stelle das gegeben zu haben, was ihm rechtmäßig zusteht. Wer den anderen mit Nächstenliebe begegnet, ist vor allem gerecht zu ihnen. Die Gerechtigkeit ist der Liebe nicht nur in keiner Weise fremd, sie ist nicht nur kein alternativer oder paralleler Weg zur ihr: Die Gerechtigkeit ist untrennbar mit der Liebe verbunden, sie ist ein ihr innewohnendes Element" (CiV 6).

In einer Ansprache an den Klerus der Diözese Rom erklärte Papst Franziskus die Gegenwart als eine „Zeit der Barmherzigkeit“. Er bezieht sich auf eine Predigt von Johannes Paul II. in der es heißt: „,das Licht der göttlichen Barmherzigkeit, das der Herr durch das Charisma von Schwester Faustyna der Welt gleichsam zurückgeben wollte, wird den Weg der Menschen des dritten Jahrtausends erhellen“30. Papst Franziskus verbindet die Barmherzigkeit mit dem Evangelisierungsauftrag sehr deutlich. Laut seiner Lehre wird die Evangelisation durch „einen unerschöpflichen Wunsch, Barmherzigkeit anzubieten - eine Frucht der eigenen Erfahrung der unendlichen Barmherzigkeit des himmlischen Vaters und ihrer Tragweite“ motiviert (vgl. EG 24). Er wünscht sich eine Kirche, die „der Ort der ungeschuldeten Barmherzigkeit" ist, „wo alle sich aufgenommen und geliebt fühlen können, wo sie Verzeihung erfahren und sich ermutigt fühlen können, gemäß dem guten Leben des Evangeliums zu leben“ (EG 114). Zum Wesen der Kirche gehört sowohl ihre missionarische Aufgabe als auch ihr Dienst an den Armen. „Wie die Kirche von Natur aus missionarisch ist - lehrt Franziskus - so entspringt aus dieser Natur zwangsläufig die wirkliche Nächstenliebe, das Mitgefühl, das versteht, beisteht und fördert" (EG 179).

Um die ,Zeit der Barmherzigkeit“" noch mehr zum Ausdruck zu bringen, ruft Franziskus „das außerordentliche Jubiläum der Barmherzigkeit“ aus. In seinem Schreiben Misericordiae vultus begründet er seine Entscheidung mit folgenden Worten: „Es gibt Augenblicke, in denen wir aufgerufen sind, in ganz besonderer Weise den Blick auf die Barmherzigkeit zu richten und dabei selbst zum wirkungsvollen Zeichen des Handelns des Vaters zu werden. Genau darum habe ich ein außerordentliches Jubiläum der Barmherzigkeit ausgerufen. Es soll eine Zeit der Gnade für die Kirche sein und helfen, das Zeugnis der Gläubigen stärker und wirkungsvoller zu machen“31.

\footnotetext{
${ }^{30}$ Vgl. Franziskus, Die Ansprache an den Klerus der Diözese Rom 06.03.2014. Text: https:// w2.vatican.va/content/francesco/de/speeches/2014/march/documents/papa-francesco_20140306_cle ro-diocesi-roma.html [zugriff am 06.07.2015].

${ }^{31}$ Franziskus, Verkündigungsbulle Misericordiae vultus zum Außerordentlichen Jubiläum der Barmherzigkeit, deutsche Übersetzung, Verlautbarungen des apostolischen Stuhls Bd. 200, hrsg. Sekretariat der Deutschen Bischofskonferenz, Bonn 2015, Nr. 3 (ab hier: MV).
} 
Die Barmherzigkeit ist kein Nebenthema der kirchlichen Lehre des letzten Jahrzehnts, sondern wahrlich ein grundlegendes Thema. Als Antwort auf die gegenwärtigen „Zeichen der Zeit“ bildet sie das Grundparadigma der ganzen Pastoral der Kirche. Sehr deutlich bringt das Franziskus in folgenden Worten zum Ausdruck: „Der Tragebalken, der das Leben der Kirche stützt, ist die Barmherzigkeit. Ihr gesamtes pastorales Handeln sollte umgeben sein von der Zärtlichkeit, mit der sie sich an die Gläubigen wendet; ihre Verkündigung und ihr Zeugnis gegenüber der Welt können nicht ohne Barmherzigkeit geschehen. Die Glaubwürdigkeit der Kirche führt über den Weg der barmherzigen und mitleidenden Liebe“"32.

\section{Prinzipien und Bereiche der Pastoral der Barmherzigkeit}

Eine Pastoral der Barmherzigkeit kann nicht von einem praktischen Standpunkt ausgehen. Sie muss sich nämlich auf eine Spiritualität der Barmherzigkeit, eine „Mystik des Erbarmens“ ${ }^{\text {(33 }}$ stützen. In diesem Kontext lassen sich die Papstworte aus dem Apostolischen Schreiben Novo millennio ineunte, die Joahennes Paul II. in Bezug auf die „Spiritualität der Gemeinschaft" formulierte, so interpretieren: Was bedeutet das konkret, dass die Barmherzigkeit ein Grundprinzip der Pastoral ist? Hier könnte die Rede sofort praktisch werden, doch es wäre falsch, einem solchen Anstoß nachzugeben. Vor der Planung konkreter Initiativen gilt es, eine Spiritualität der Barmherzigkeit zu fördern, indem man sie überall dort als Erziehungsprinzip herausstellt, wo man den Menschen und Christen formt, wo man die geweihten Amtsträger, die Ordensleute und die Mitarbeiter in der Seelsorge ausbildet, wo man die Familien und Gemeinden aufbaut ${ }^{\star 34}$.

Die Spiritualität der Barmherzigkeit bzw. die ,neue pastorale Mentalität“ oder der „,neue pastorale Still“ sollen auf einigen Prinzipien gebaut werden. Zuerst geht es hier um das Prinzip des Primats der Gnade, das ein „Leuchtfeuer“" sein muss, das die Überlegungen zur Evangelisierung und Pastoral ständig erhellt (vgl. EG 112). Die Grundüberzeugung ,führt zu einem neuen Stil der Seelsorge, einer Seelsorge, die auf den Geist Gottes vertraut; einer Seelsorge, die im Hinblick auf Gottes eigene Wege gelassen sein kann; einer Seelsorge, die ihren engagierten Mitarbeiterinnen und Mitarbeitern Freude und Entlastung bringt. Denn Gott ist der eigentliche Seelsorger" ${ }^{\text {"35. }}$.

${ }^{32}$ Ebd. Nr. 10.

${ }^{33}$ P.M. Zulehner, Gott ist größer als unser Herz, op. cit., S. 158-160.

${ }^{34}$ Vgl. Johannes Paul II., Apostolisches Schreiben Novo millennio ineunte, deutsche Übersetzung, Verlautbarungen des Apostolischen Stuhls Bd. 150, hrsg. Sekretariat der Deutschen Bischofskonferenz, Bonn 2001, Nr. 43 (ab hier: NMI).

${ }^{35}$ B. Elbs, Im Stallgeruch der Schafe. Wege pastoraler Arbeit im 3. Jahrtausend, Wien-Graz-Klagenfurt 2014, S. 192. 
Dann muss die Methode der Begegnung und des Dialoges berücksichtigt werden ${ }^{36}$. Der Dialog ist die entscheidende Form der Pastoral, ,um mit den Menschen von heute in der Welt von heute in Verbindung zu treten“37. In der dialogischen pastoralen Begegnung spielt eine „Kunst des Zuhörens“ eine wichtige Rolle. „Nur auf der Grundlage dieses achtungsvollen, mitfühlenden Zuhörens ist es möglich, die Wege für ein echtes Wachstum zu finden, das Verlangen nach dem christlichen Ideal und die Sehnsucht zu wecken, voll auf die Liebe Gottes $\mathrm{zu}$ antworten und das Beste, das Gott im eigenen Leben ausgesät hat, zu entfalten“ (EG 171). Die Kunst des Zuhörens verlangt nach der „Fähigkeit des Herzens", die in der Verständigung mit dem anderen eine große Rolle spielt. Sie ermöglicht die wahre geistliche Begegnung und befähigt dazu, ,die passende Geste und das passende Wort zu finden, die uns aus der bequemen Position des $\mathrm{Zu}$ schauers herausholen“" (EG 171) ${ }^{38}$.

Nicht weniger wichtig ist eine „Kunst des Wartens und der Geduld“. Diese Fähigkeiten gehören zu einer „Pädagogik, welche die Personen schrittweise zur vollen Aneignung des Mysteriums hinführt“ (EG 171). Diese Pädagogik gehört zum erneuerten pastoralen Stil, der sich in einer „Kunst der Begleitung” ausdrückt. Die Kirche muss alle, die in ihrer pastoralen Tätigkeit das Prinzip der Barmherzigkeit berücksichtigen wollen, lehren, ,,vor dem heiligen Boden des anderen sich die Sandalen von den Füßen zu streifen (vgl. Ex 3,5)“ (EG 169). Solche Kunst des Wartens und der Geduld stützt sich auf dem fundamentalen pastoralen Prinzip der Freiheit, weil die Kirche die Wahrheit nicht aufzwingen darf und nur an die Freiheit appellieren muss (vgl. EG 165).

Die Pastoral der Barmherzigkeit kann nicht auf die materielle Unterstützung der Armen reduziert werden. Sie umfasst alle Bereiche der Selbstverwirklichung der Kirche: Verkündigung, Liturgie und Diakonie. Das erste Werk der Barmherzigkeit ist die Verkündigung des Evangeliums. Eine pastorale Option für die Armen soll sich grundsätzlich in der religiösen Zuwendung zeigen. „Die schlimmste Diskriminierung, unter der die Armen leiden, ist der Mangel an geistlicher Zuwendung“ (EG 200). Die Verkündigung der Barmherzigkeit Gottes als „das pulsierende Herz des Evangeliums" stellt Franziskus in seinem Schreiben für das „Jahr der Barmherzigkeit“" als den bevorzugten Auftrag der Kirche hin. Durch diese Verkündigung soll die Barmherzigkeit das Herz und den Verstand der Menschen erreichen (Vgl. MV 12).

Im Bereich Liturgie geht es in der Pastoral der Barmherzigkeit darum, „die Menschen zu den Quellen des Erbarmens des Heilands" zu führen, welche die Kir-

${ }^{36}$ P. Bacq, Für eine Erneuerung vom Ursprung her. Auf dem Weg zu einer ,,zeugenden Pastoral ", in: Frei geben. Pastoraltheologische Impulse aus Frankreich, hrsg. R. Feiter, H. Müller, übersetzt von H. Müller, W. Rauscher, Ostfildern 2012, S. 45-49.

${ }^{37}$ Vgl. B. Elbs, Im Stallgeruch der Schafe, op. cit., S. 184-185.

${ }^{38}$ Vgl. P.M. Zulehner, Ein neues Pfingsten. Ermutigung zu einem Weg der Hoffnung, Ostfildern 2008, S. 75-78. 
che hütet und aus denen sie austeilt. Eine große Bedeutung kommt in diesem Zusammenhang der ständigen Betrachtung des Wortes Gottes und vor allem der bewussten, mit innerer Reife vollzogenen Feier der Eucharistie und des Sakraments der Buße und der Versöhnung zu (vgl. DiM 13). Diese Aufgabe der Pastoral des Erbarmens setzt das Vertrauen in die pastorale Effektivität und die geistige Kraft „,der armen Mittel“, die der Kirche von Jesus anvertraut sind.

Die kirchliche Verkündigung des Evangeliums der Barmherzigkeit wäre jedoch sinnlos, wenn sie durch die Werke der Barmherzigkeit nicht bestätigt würde, weil „die Liebe der Werke verleiht der Liebe der Worte eine unmissverständliche Kraft" (NMI 50). Für die kirchliche Diakonie sind zwei grundsätzlichen Prinzipien der Pastoral der Barmherzigkeit zu nennen. Erstens: die Barmherzigkeit ist keine zeitlich und räumlich begrenzte Aktivität der Christen sondern ,ein Lebensstil, ein wesentliches und immerwährendes Kennzeichen der christlichen Berufung" (vgl. DiM 14). Zweitens: Die christliche erbarmende Liebe hat nicht als Ziel den Armen die bloße materielle bzw. geistige Unterstützung zu erweisen, sondern mit ihnen in communio zu treten. „Unser Einsatz - so Papst Franziskus - besteht nicht ausschließlich in Taten oder in Förderungs- und Hilfsprogrammen; was der Heilige Geist in Gang setzt, ist nicht ein übertriebener Aktivismus, sondern vor allem eine aufmerksame Zuwendung zum anderen, indem man ihn als eines Wesens mit sich selbst betrachtet" (EG 199). Nicht die Hilfe für die Armen, sondern die Liebe zu ihnen unterscheidet „die authentische Option für die Armen von jeder Ideologie, von jeglicher Absicht, die Armen zugunsten persönlicher oder politischer Interessen zu gebrauchen. Nur das macht es möglich, »dass sich die Armen in jeder christlichen Gemeinde wie »zu Hause« fühlen“ (EG 199).

\section{Schlussbemerkung}

Eine kompakte Beschreibung der Pastoral der Barmherzigkeit gab Papst Franziskus in einer seinen Ansprachen an den römischen Klerus ${ }^{39}$. In ihr stellt er fest, dass die Pastoral der Barmherzigkeit ,weder Nachsichtigkeit noch große Strenge" bedeutet. Weder der Anhänger des Laxismus noch der Rigorist legt Zeugnis ab von der Barmherzigkeit Gottes. „Der Rigorist zieht sich aus der Affäre: denn er fesselt die Person an das kalt und streng aufgefasste Gesetz. Auch der Anhänger des Laxismus zieht sich aus der Affäre: Er ist nur scheinbar barmherzig, aber in Wirklichkeit nimmt er das Problem jenes Gewissens nicht ernst, indem er die Sünde herunterspielt“. Die Pastoral der Barmherzigkeit nimmt sich der Peron an, übernimmt die Verantwortung für sie, hört ihr aufmerksam zu, nähert sich der Situation mit Respekt und mit Wahrheit und begleitet sie auf dem Weg der Versöhnung. Die Grundlage einer solchen Pastoral bildet „die Mystago-

\footnotetext{
${ }^{39}$ Franziskus, Die Ansprache an den Klerus der Diözese Rom 06.03.2014, op. cit.
} 
gie des offenen Herzens und der offenen Augen“40 bzw. „die Mystik des Erbarmens“41. „Die Grundsymphonie einer Mystik des Erbarmens kennt viele Sätze und Variationen. Ihr Ziel ist immer, dass Gottes Wesen das Wesen des Menschen zuinnerst durchfließt und damit formt. Das macht es dem Menschen möglich, Gottes Erbarmen in der Welt »gleichsam« zu sein, es sichtbar zu machen in seinem Denken und Tun“42.

\section{Summary}

The pastoral care of the church needs a paradigm, on which it can build its practice. This paradigm must take signs of the contemporary times into account. The message of mercy was crucial for the pontificates of John XXIII, John Paul II and Benedict XVI. Mercy is also an essential theme of teaching for Pope Francis. The primary sign of the contemporary times is mercy. At times we are called to gaze even more attentively on mercy so that we may become a more effective sign of the Father's action in our lives (Pope Francis). The main issue of the present article is mercy as a paradigm of the missionary and pastoral care of the church. Three statements create the issues for this study: pastoral ministry in a missionary key, contemporary times as the times of mercy and principles and areas of the pastoral of the mercy.

\section{Keywords}

mercy, pastoral care, new evangelisation, missionary conversion, signs of the times

\section{Słowa kluczowe}

miłosierdzie, duszpasterstwo, nowa ewangelizacja, misyjne nawrócenie, znaki czasu

\section{Literatur}

Bacq P., Für eine Erneuerung vom Ursprung her. Auf dem Weg zu einer „zeugenden Pastoral", in: Frei geben. Pastoraltheologische Impulse aus Frankreich, hrsg. R. Feiter, H. Müller, übersetzt von H. Müller, W. Rauscher, Ostfildern 2012, S. 30-55.

Bajda J., Encyklika o Bożym miłosierdziu, „Collectanea Theologica” 4 (1981), S. 85-88.

Benedikt XVI, Enzyklika Deus caritas est über die christliche Liebe (25.12.2005), deutsche Übersetzung, Verlautbarungen des apostolischen Stuhls Bd. 171, hrsg. Sekretariat der Deutschen Bischofskonferenz, Bonn 2006.

Benedikt XVI, Enzyklika Caritas in veritate über die ganzheitliche Entwicklung des Menschen in der Liebe und in der Wahrheit (29.06.2009), deutsche Übersetzung, Verlautbarungen des apostolischen Stuhls Bd. 186, hrsg. Sekretariat der Deutschen Bischofskonferenz, Bonn 2009.

${ }^{40}$ M. Polak, Pedagogia ewangelizacji na przedpolach wiary, in: Peryferie wiary wyzwaniem dla Kościoła, hrsg. W. Przygoda, M. Fiałkowski, Lublin 2015, S. 79-81.

${ }^{41}$ P.M. Zulehner, Gott ist größer als unser Herz, op. cit., S. 160.

${ }^{42}$ Ebd. 
Die Deutsche Bischofskonferenz, „Zeit zur Aussaat“. Missionarisch Kirche sein (26.11. 2000), Verlautbarungen der deutschen Bischofskonferenz, hrsg. Sekretariat der deutschen Bischofskonferenz, Bd. 68, Bonn 2000.

Elbs B., Im Stallgeruch der Schafe. Wege pastoraler Arbeit im 3. Jahrtausend, Wien-Graz-Klagenfurt 2014.

Franziskus, Apostolisches Schreiben Evangelii gaudium über die Verkündigung des Evangeliums in der Welt von heute (24.11.2013), deutsche Übersetzung, Verlautbarungen des Apostolischen Stuhls, hrsg. Sekretariat der deutschen Bischofskonferenz, Bd. 194, Bonn 2014.

Franziskus, Verkündigungsbulle Misericordiae vultus zum Außerordentlichen Jubiläum der Barmherzigkeit, deutsche Übersetzung, Verlautbarungen des apostolischen Stuhls Bd. 200, hrsg. Sekretariat der Deutschen Bischofskonferenz, Bonn 2015.

Franziskus, Die Ansprache an den Klerus der Diözese Rom 06.03.2014. Text: https://w2. vatican.va/content/francesco/de/speeches/2014/march/documents/papa-francesco_2014 0306_clero-diocesi-roma.html [dostęp: 06.07.2015].

Gajda A., Miłosierdzie większe niż sprawiedliwość. Piękno myśli i słowa $w$ encyklice „Dives in missericordia”, „Zeszyty Naukowe KUL” 3 (2004), S. 85-101.

Grzybek S., Jaworski M., Encyklika Ojca Świętego Jana Pawła II o Bożym miłosierdziu „Dives in missericordia”. Tekst i komentarz, Kraków 1981.

Jan Pawet II - „,Dives in missericordia”. Tekst i komentarze, hrsg. S. Nagy, Lublin 1983.

Johannes Paul II, Enzyklika Dives in missericordia über das göttliche Erbarmen (30.11.1980), deutsche Übersetzung, Verlautbarungen des Apostolischen Stuhls, hrsg. Sekretariat der deutschen Bischofskonferenz, Bd. 26, Bonn 1980.

Johannes Paul II., Enzyklika Redemptoris missio über die fortdauernde Gültigkeit des missionarischen Auftrages (07.12.1990), deutsche Übersetzung, Verlautbarungen des Apostolischen Stuhls, hrsg. Sekretariat der deutschen Bischofskonferenz, Bd. 100, Bonn 1991.

Johannes Paul II., Enzyklika Redemptoris missio über die fortdauernde Gültigkeit des missionarischen Auftrages (07.12.1990), deutsche Übersetzung, Verlautbarungen des Apostolischen Stuhls, hrsg. Sekretariat der deutschen Bischofskonferenz, Bd. 100, Bonn 1991.

Johannes Paul II., Apostolisches Schreiben Novo millennio ineunte, deutsche Übersetzung, Verlautbarungen des Apostolischen Stuhls Bd. 150, hrsg. Sekretariat der Deutschen Bischofskonferenz, Bonn 2001.

Johannes Paul II, Barmherzigkeit Gottes - Quelle der Hoffnung, ausgewählt und eingeleitet von E. Olk, Einsideln 2011.

Johannes Paul II, Erinnerung und Identität, deutsche Übersetzung, Augsburg 2005.

Johannes Paul II, Predigt bei der Heiligsprechung von Maria Faustyna Kowalska Lagiewniki 30.04.2000, Text: http://w2.vatican.va/content/john-paul-ii/de/homilies/2000/docu ments/hf_jp-ii_hom_20000430_faustina. html [dostęp: 03.07.2015].

Kasper W., Barmherzigkeit. Grundbegriff des Evangeliums - Schlüssel christlichen Lebens, Freiburg im Breisgau 2012. 
Knobloch S., Ungenutztes Potential. Zwischen religiöser Sehnsucht und Kirchenkrise, Ostfildern 2011.

XIII. Ordentliche Vollversammlung der Bischofssynode, Neuevangelisierung für die Weitergabe des Glaubens, Lineamenta in: http://www.vatican.va/roman_curia/synod/doc uments/rc_synod_doc_20110202_lineamenta-xiii-assembly_ge.html [dostęp: 15.06.2015]

Paul VI., Apostolisches Schreiben Evangelii nuntiandi über die Evangelisierung in der Welt von heute (8.12.1975), deutsche Übersetzung, Verlautbarungen des Apostolischen Stuhls, hrsg. Sekretariat der deutschen Bischofskonferenz, Bd. 2, Bonn 1975.

Polak M., Duszpasterstwo miłosierdzia. Miłosierdzie jako paradygmat pastoralnej misji Kościoła, „Teologia Praktyczna” 15 (2014), S. 7-21.

Polak M., Pedagogia ewangelizacji na przedpolach wiary, in: Peryferie wiary wyzwaniem dla Kościoła, hrsg. W. Przygoda, M. Fiałkowski, Lublin 2015, S. 61-82.

Ratzinger J., Predigt während der Messe pro eligendo romano Pontifice. Patriarchalbasilika St. Peter 18.04.2005. Text: http://www.vatican.va/gpII/documents/homily-pro-elig endo-pontifice_20050418_ge.html [dostęp: 03.07.2015].

Wobec tajemnicy Bożego Miłosierdzia, hrsg. L. Balter, Seria: Powołanie człowieka, Poznań 1991.

Zulehner P.M., Gott ist größer als unser Herz. Eine Pastoral des Erbarmens, Ostfildern 2006.

Zulehner P.M., Kirchenvisionen. Orientierung in Zeiten des Kirchenumbaus, Ostfildern 2012.

Zulehner P.M., Ein neues Pfingsten. Ermutigung zu einem Weg der Hoffnung, Ostfildern 2008. 